\title{
Sum-rule constraints on possible diphoton resonances at LHC
}

\author{
P. Roig ${ }^{1, a}$ and J.J. Sanz-Cillero $2, b$ c \\ ${ }^{1}$ Departamento de Física, Centro de Investigación y de Estudios Avanzados del Instituto Politécnico Na- \\ cional, Apartado Postal 14-740, 07000 México City, México \\ ${ }^{2}$ Departamento de Física Teórica I, Universidad Complutense de Madrid, E-28040 Madrid, Spain
}

\begin{abstract}
The study of the forward scattering amplitude $V(k, \lambda) V\left(k^{\prime} \lambda^{\prime}\right) \rightarrow$ $V(k, \lambda) V\left(k^{\prime}, \lambda^{\prime}\right)$ of real massless gauge bosons $V$, e.g. photons or gluons, leads to a sumrule that can be used to investigate beyond the Standard Model signals at LHC in the $\gamma \gamma$ channel. The sum-rule only relies on general properties such as analyticity, unitarity and crossing. We use the now buried "750 GeV diphoton resonance" as a case of study to exemplify the constraints that the forward sum-rule requires to any new physics candidate. In the case of a large $\gamma \gamma$ or $g g$ partial width, of the order of $10 \mathrm{GeV}$ in our $750 \mathrm{GeV}$ analysis, one finds that an infinite tower of states with spin $J_{R}=2$ and higher must be ultimately incorporated to the beyond Standard Model theory in order to fulfill the sumrule. We expect these techniques may be useful in next diphoton searches at LHC and future colliders.
\end{abstract}

\section{Introduction}

On December 2015, the $13 \mathrm{TeV}$ integrated luminosity of $3.2 \mathrm{fb}^{-1}$ and $2.6 \mathrm{fb}^{-1}$ in ATLAS and CMS, respectively, showed an excess in the diphoton spectrum, hinting the presence of a resonance with invariant mass around $750 \mathrm{GeV}$ [1]. The expectancy grew on March 2016, at Rencontres de Moriond, after reanalyses of ATLAS and CMS data from December 2015, together with the recovery and study of additional $0.6 \mathrm{fb}^{-1} \mathrm{CMS}$ (at $0 \mathrm{~T}$ ) [2]. ATLAS showed the highest significance and pushed the production cross section $\sigma(p p \rightarrow R \rightarrow \gamma \gamma)$ up, with the combined value of $4.2 \pm 2 \mathrm{fb}(4.2 \pm 2.6 \mathrm{fb})$ for the possible spin-0 (spin-2) diphoton resonance at $750 \mathrm{GeV} \mathrm{[3].} \mathrm{The} \mathrm{best} \mathrm{fit} \mathrm{preferred} \mathrm{a} \mathrm{broad}$ total width of $\sim 45 \mathrm{GeV}$ [3] and some works pointed out $O(\mathrm{GeV})$ partial widths for $R \rightarrow g g$ and $R \rightarrow \gamma \gamma[5,6]$, though with huge uncertainties. These features were difficult to be fulfilled for weakly-coupled beyond Standard Model (BSM) theories, where the assumed perturbativity broke down around the TeV region $[4,7])$. Thus, Strongly-coupled models and their higher dimensional duals seemed to be favoured [8-12].

However, by August 2016, ATLAS and CMS accumulated $12.9 \mathrm{fb}^{-1}$ and $15.4 \mathrm{fb}^{-1}$ data, respectively, showing essentially no significant excess around $750 \mathrm{GeV}$ [13]. Even though the "750 GeV diphoton resonance" is now buried this does not mean the end of searches in the diphoton channel. From this perpective, the theoretical constraints of the analysis [14] this talk is based on may be useful

\footnotetext{
ae-mail: proig@fis.cinvestav.mx

be-mail: jjsanzcillero@ucm.es

${ }^{\mathrm{c}}$ Speaker.
} 


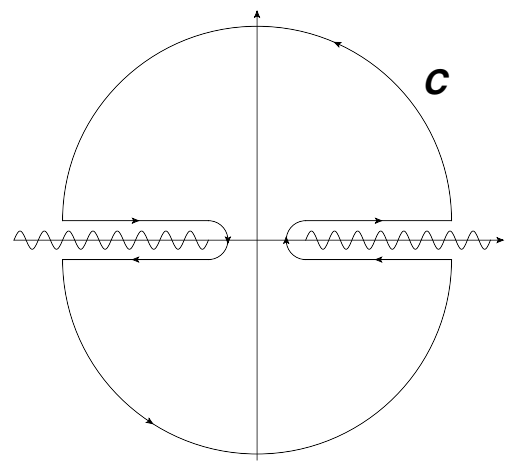

Figure 1. Integration contour for the sum-rule complex integral.

in future scans: a lone scalar resonance with a large diphoton partial width leads to inconsistencies with the basic assumptions of unitarity, crossing and analyticity. These issues can be only solved in two ways: 1) the appearance of states with spin $J_{R} \geq 2$ (expected in composite BSM theories or higher dimensional duals of strongly coupled field theories) [8-12]; 2) the breakdown of perturbation theory around the $\mathrm{TeV}[4,7]$.

These proceedings are organized as follows: In Sec. 2 we derive the forward sum-rule (FSR) that constrains the scattering of real spin-1 particles. We then study in Sec. 3 the impact of a scalar resonance, e.g. produced in the diphoton channel, on the FSR. One finds an unbalance in the FSR which requires the presence of additional contributions, where a spin-2 resonance appears as a potential candidate. We note that the sum-rule studied here only relies on unitarity, analyticity and crossing symmetry and is therefore fulfilled in any possible BSM extension that assumes these general properties. Finally in Sec. 4 we provide some numerical estimates of what kind of $J_{R}=2$ resonance signal one could expect if a spin-0 resonance shows up in LHC's diphoton spectrum, where we take the "750 GeV resonance" as a case of study to exemplify the analysis. Some conclusions are gathered in Sec. 5.

\section{Theoretical framework: forward sum-rules for spin-1 particle scattering}

Let us consider a spin-1 particle $V(k, \lambda)$ with momentum $k$ and helicity $\lambda= \pm 1$. The particle $V$ is assumed to be described by a real field in quantum field theory (QFT). In principle it may carry other group indices, but this will not be relevant for the derivation of the sum-rule, only for some details of the later low-energy matching. Examples of this type of particles $V$ would be the photon $\gamma$ or the gluon $g^{a}$ (for a fixed gluon colour index $a$, not averaged or summed).

Ref. [14] studied the forward collision $(t=0), T_{\Delta \lambda}$, as a function of the kinematical variable $v \equiv(s-u) / 2,{ }^{1}$

$$
T_{\Delta \lambda}(v)=T\left(V(k, \lambda) V\left(k^{\prime}, \lambda^{\prime}\right) \rightarrow V(k, \lambda) V\left(k^{\prime}, \lambda^{\prime}\right)\right) .
$$

with helicity difference $\Delta \lambda=\left|\lambda-\lambda^{\prime}\right|$. For instance, the case $V=\gamma$ corresponds to the forward scattering $\gamma(k, \lambda) \gamma\left(k^{\prime} \lambda^{\prime}\right) \rightarrow \gamma(k, \lambda) \gamma\left(k^{\prime} \lambda^{\prime}\right)$.

We now proceed to impose the three basic ingredients of the sum-rule:

\footnotetext{
${ }^{1}$ The use of the kinematical variable $v \equiv(s-u) / 2$ is customary in fixed- $t$ dispersive analyses of scattering amplitudes with definite $s \leftrightarrow u$ crossing properties (see in general Ref. [15]). In particular, for $t=0$ one has $v=s$.
} 
1. Analyticity: In Fig. 1 one can see the analytical structure of the function $T_{\Delta \lambda}(v)$ in the complex $v$-plane. There is a right-hand absorptive cut that corresponds to the intermediate on-shell states when $s>0$ (s-channel cut) and a left-hand cut related to the intermediate production of on-shell states when $u>0$ (u-channel cut). This allows us to write down the Cauchy integral at the close contour $C$ in Fig. 1,

$$
\frac{1}{2 \pi i} \oint_{C} \mathrm{~d} v^{\prime} \frac{T_{\Delta \lambda}\left(v^{\prime}\right)}{v^{\prime}\left(v^{\prime}-v\right)}=\frac{T_{\Delta \lambda}(v)}{v}+\frac{T_{\Delta \lambda}(0)}{(-v)},
$$

taking $v$ any complex value within the contour $C$. In the right-hand side (rhs) we have used Cauchy's theorem and the fact that the in the first Riemann sheet, where this integral is performed, $T_{\Delta \lambda}\left(v^{\prime}\right)$ is analytical within the contour $C$ and the only poles are at $v^{\prime}=0$ and $v^{\prime}=v$ due to the denominator in the integrand.

2. Unitarity: scattering cross sections are limited by the Froissart bound [16]. This implies constraints on the scattering amplitude $T\left(V(k, \lambda) V\left(k^{\prime} \lambda^{\prime}\right) \rightarrow X\right)$ for any final product $X$ and, in particular, on the forward scattering amplitude in the form

$$
\left|T_{\Delta \lambda}(v)\right|^{2}<C v \ln ^{2}\left(v / v_{0}\right),
$$

for some constants $C, v_{0}$. This means that the integral in Eq. (2) over the external circular parts of the contour $C$ vanishes when its radius goes to infinity and the once-subtracted integrals on the real axis converge:

$$
\begin{aligned}
& T_{\Delta \lambda}(v)-T_{\Delta \lambda}(0)=\frac{v}{2 \pi i} \oint_{C} \mathrm{~d} v^{\prime} \frac{T_{\Delta \lambda}\left(v^{\prime}\right)}{v^{\prime}\left(v^{\prime}-v\right)} \\
& =\frac{v}{2 \pi i} \int_{-\infty}^{v_{\mathrm{th}}^{(\mathrm{u})}} \mathrm{d} v^{\prime} \frac{\left(T_{\Delta \lambda}\left(v^{\prime}+i \epsilon\right)-T_{\Delta \lambda}\left(v^{\prime}-i \epsilon\right)\right)}{v^{\prime}\left(v^{\prime}-v\right)}+\frac{v}{2 \pi i} \int_{v_{\mathrm{th}}^{(\mathrm{s})}}^{\infty} \mathrm{d} v^{\prime} \frac{\left(T_{\Delta \lambda}\left(v^{\prime}+i \epsilon\right)-T_{\Delta \lambda}\left(v^{\prime}-i \epsilon\right)\right)}{v^{\prime}\left(v^{\prime}-v\right)},
\end{aligned}
$$

with $v_{\mathrm{th}}^{(\mathrm{s})}$ and $v_{\mathrm{th}}^{(\mathrm{u})}$ the threshold of the right-hand and left-hand cuts, respectively. Both thresholds are related below through crossing symmetry. By the analyticity Schwartz reflection principle one can rewrite $T_{\Delta \lambda}\left(v^{\prime}-i \epsilon\right)=T_{\Delta \lambda}\left(v^{\prime}+i \epsilon\right)^{*}$ :

$$
T_{\Delta \lambda}(v)-T_{\Delta \lambda}(0)=\frac{v}{\pi} \int_{-\infty}^{v_{\mathrm{th}}^{(\mathrm{u})}} \mathrm{d} v^{\prime} \frac{\operatorname{Im} T_{\Delta \lambda}\left(v^{\prime}+i \epsilon\right)}{v^{\prime}\left(v^{\prime}-v\right)}+\frac{v}{\pi} \int_{v_{\mathrm{th}}}^{\infty} \mathrm{d} v^{\prime} \frac{\operatorname{Im} T_{\Delta \lambda}\left(v^{\prime}+i \epsilon\right)}{v^{\prime}\left(v^{\prime}-v\right)},
$$

where we have used that $2 i \operatorname{Im} T_{\Delta \lambda}\left(v^{\prime}+i \epsilon\right)=T_{\Delta \lambda}\left(v^{\prime}+i \epsilon\right)-T_{\Delta \lambda}\left(v^{\prime}+i \epsilon\right)^{*}$.

3. Crossing symmetry: Let us consider a forward amplitude with definite helicity, e.g, $T\left(V(k,+) V\left(k^{\prime},+\right) \rightarrow V(k,+) V\left(k^{\prime},+\right)\right)$. If one now exchanges the incoming and outgoing real vector $V$ with momentum $k^{\prime}$ in the latter example, crossing symmetry tells us that the previous amplitude coincides with that for $T\left(V(k,+) V\left(-k^{\prime},-\right) \rightarrow V(k,+) V\left(-k^{\prime},-\right)\right)$, which corresponds to the replacements $k^{\prime} \rightarrow-k^{\prime}, \lambda^{\prime} \rightarrow-\lambda^{\prime}$ and $v \rightarrow-v$. Hence, in general one has the relation

$$
T_{\Delta \lambda}(v)=T_{\overline{\Delta \lambda}}(-v),
$$

with $\overline{\Delta \lambda}=2-\lambda$. The first implication of this crossing relation is that the left-hand and righthand thresholds are related in the form $v_{\text {th }}^{(\mathrm{s})}=-v_{\text {th }}^{(\mathrm{u})} \equiv v_{\mathrm{th}}$. This implies that in the previous integral (4) one can rewrite the left-hand cut as a right-hand cut integral in the way

$$
T_{\Delta \lambda}(v)-T_{\Delta \lambda}(0)=\frac{v}{\pi} \int_{v_{\text {th }}}^{\infty} \mathrm{d} v^{\prime}\left(-\frac{\operatorname{Im} T_{\overline{\Delta \lambda}}\left(v^{\prime}+i \epsilon\right)}{v^{\prime}\left(v^{\prime}+v\right)}+\frac{\operatorname{Im} T_{\Delta \lambda}\left(v^{\prime}+i \epsilon\right)}{v^{\prime}\left(v^{\prime}-v\right)}\right) .
$$


This equation provides the master relation for the sum-rule studied in Ref. [14] and the RoyGerasimov-Moulin sum-rule for the inclusive $\gamma \gamma$ cross section $\sigma_{\Delta \lambda}=\sigma\left(\gamma(k, \lambda) \gamma\left(k^{\prime}, \lambda^{\prime}\right) \rightarrow X\right)$ [17]. Its $\mathrm{n}$-th derivative evaluated at $v=0$ yields

$$
\frac{1}{n !} \frac{\mathrm{d}^{\mathrm{n}}}{\mathrm{d} v^{\mathrm{n}}} T_{\Delta \lambda}(0)=\frac{1}{\pi} \int_{v_{\mathrm{th}}}^{\infty} \frac{\mathrm{d} v^{\prime}}{v^{\prime} n+1}\left((-1)^{n} \operatorname{Im} T_{\overline{\Delta \lambda}}\left(v^{\prime}+i \epsilon\right)+\operatorname{Im} T_{\Delta \lambda}\left(v^{\prime}+i \epsilon\right)\right),
$$

for $n \geq 1$.

In the case when $V$ is a massless abelian gauge boson like, e.g., the photon, the low-energy forward scattering is provided by the Euler-Heisenberg Lagrangian [18]. The effective field theory (EFT) operators contributing to this process have dimesion 8 or higher and, therefore, the low-energy amplitude behaves at $v \rightarrow 0$ like

$$
T_{\Delta \lambda}(v) \stackrel{v \rightarrow 0}{=} c_{\Delta \lambda} v^{2}+O\left(v^{3}\right) .
$$

Thus, since $T_{\Delta \lambda}^{\prime}(0)=0$, Eq. (8) for $n=1$ implies the forward sum-rule in [14],

$$
0=\frac{1}{\pi} \int_{v_{\mathrm{th}}}^{\infty} \frac{\mathrm{d} v^{\prime}}{\left(v^{\prime}\right)^{2}}\left(\operatorname{Im} T_{2}\left(v^{\prime}+i \epsilon\right)-\operatorname{Im} T_{0}\left(v^{\prime}+i \epsilon\right)\right) .
$$

It is equivalent to the Roy-Gerasimov-Moulin sum-rule for the inclusive $\gamma \gamma$ cross section $\sigma_{\Delta \lambda}=\sigma\left(\gamma(k, \lambda) \gamma\left(k^{\prime}, \lambda^{\prime}\right) \rightarrow X\right)[17]:$

$$
0=\frac{1}{\pi} \int_{v_{\mathrm{th}}}^{\infty} \frac{\mathrm{d} v^{\prime}}{v^{\prime}}\left[\sigma_{2}\left(v^{\prime}\right)-\sigma_{0}\left(v^{\prime}\right)\right]
$$

by means of the relation $\sigma_{\Delta \lambda}\left(v^{\prime}\right)=\operatorname{Im} T_{\Delta \lambda}\left(v^{\prime}+i \epsilon\right) / v^{\prime}$.

Eq. (8) implies further sum-rules. For instance, for even $n$ the right-hand side integral is positivedefinite and one has $d^{n} T_{\Delta \lambda} / d v^{n}>0$ at $v=0$. In the (abelian gauge boson) photon scattering case this means that, for instance, the corresponding combination $c_{\Delta \lambda}$ of Euler-Heisenberg low-energy constants is positive.

An analogous result can be derived for non-abelian gauge boson scattering like, e.g., the $g^{a} g^{a} \rightarrow$ $g^{a} g^{a}$ process. Around $v \sim 0$, massive states contribute to the forward scattering of two same-colour gluons through EFT operators of dimension 8 (with the colour trace of four field-strength tensors $G^{\alpha \beta}$ ) or higher. Notice that we do not discuss the general $g^{a} g^{b} \rightarrow g^{c} g^{d}$ scattering; all the initial and final gluons have the same colour $g^{a}$. Although the pure Yang-Mills (YM) Lagrangian yields no tree-level contribution to $g^{a} g^{a} \rightarrow g^{a} g^{a}$, it generates a non-vanishing forward amplitude $T_{\Delta \lambda}(v)^{\text {pure YM }}$ which starts at the loop level. Nonetheless, the pure YM theory is well behaved in the ultraviolet and fulfills Froissart's bound and the once-subtracted dispersion relation (5). For this reason, in the case of nonabelian gauge boson scattering (e.g., $\left.g^{a}(k) g^{a}\left(k^{\prime}\right) \rightarrow g^{a}(k) g^{a}\left(k^{\prime}\right)\right)$ the replacement $T_{\Delta \lambda}(v) \longrightarrow \widetilde{T}_{\Delta \lambda}(v)=$ $\left[T_{\Delta \lambda}(v)-T_{\Delta \lambda}(v)^{\text {pure } \mathrm{YM}}\right]$ is implicitly assumed in the previous equations, leading to a similar sum-rule (10) for $\widetilde{T}_{\Delta \lambda}(v)$. This result does not apply to the spontaneously broken gauge symmetry case, as physics beyond the pure YM theory can generate a contribution to the amplitude at $v \rightarrow 0$ and, hence, to the l.h.s. of the sum-rule (10). From the EFT point of view this means that there are gauge invariant operators of higher dimension that contribute to the forward amplitude and its derivative at $v=0$. In the case of the forward $Z Z$ scattering in the Standard Model $(\mathrm{SM})$, for instance, $T_{\Delta \lambda}(0), T_{\Delta \lambda}^{\prime}(0) \neq 0$ already at tree-level due to the exchange of a massive Higgs.

The contribution from neutral colourless resonances $R$ to the spectral function is given at tree-level by

$$
\left.\operatorname{Im} T_{\Delta \lambda}(v+i \epsilon)\right|_{R}=\sum_{R} 16 \pi^{2}\left(2 J_{R}+1\right) M_{R} \Gamma_{R \rightarrow[V V]_{\Delta \lambda}} \delta\left(v-M_{R}^{2}\right),
$$


and turns (10) into

$$
0=\sum_{R} 16 \pi\left(2 J_{R}+1\right) \frac{\left(\Gamma_{R \rightarrow[V V]_{2}}-\Gamma_{R \rightarrow[V V]_{0}}\right)}{M_{R}^{3}} \quad+\text { non-R, }
$$

where

$$
\Gamma_{R \rightarrow[V V]_{0}}=\Gamma_{R \rightarrow V(+) V(+)}+\Gamma_{R \rightarrow V(-) V(-)}, \Gamma_{R \rightarrow[V V]_{2}}=\Gamma_{R \rightarrow V(+) V(-)}, \Gamma_{R \rightarrow V V}=\Gamma_{R \rightarrow[V V]_{0}}+\Gamma_{R \rightarrow[V V]_{2}} .
$$

The non-R contribution represents the loop diagrams in $V V \rightarrow V V$ without an intermediate $s$ and $u$-channel resonance at tree-level.

The importance of these sum-rules relies on the fact that when the vector $V$ is massless the lowestspin resonances $\left(J_{R}=0\right)$ can only decay into $V(k, \lambda) V\left(k^{\prime}, \lambda^{\prime}\right)$ pairs with $\Delta \lambda=0$. Hence, these spin-0 resonances give a negative contribution to the sum-rule (12). The positive terms with $\Gamma_{R \rightarrow[V V]_{2}}$ only appear for higher spin resonances with $J_{R} \geq 2$ [17, 19-21]:

$$
\begin{array}{rlll}
\Gamma_{R \rightarrow V V}=\Gamma_{R \rightarrow[V V]_{0}} \geq 0, \Gamma_{R \rightarrow[V V]_{2}}=0 & \text { for } J_{R}=0 & \longrightarrow & \left(\Gamma_{R \rightarrow[V V]_{2}}-\Gamma_{R \rightarrow[V V]_{0}}\right) \leq 0, \\
\Gamma_{R \rightarrow V V}=\Gamma_{R \rightarrow[V V]_{0}}=\Gamma_{R \rightarrow[V V]_{2}}=0 & \text { for } J_{R}=1 & \longrightarrow & \left(\Gamma_{R \rightarrow[V V]_{2}}-\Gamma_{R \rightarrow[V V]_{0}}\right)=0, \\
\Gamma_{R \rightarrow[V V]_{0}} \geq 0, \quad \Gamma_{R \rightarrow[V V]_{2}} \geq 0 & \text { for } J_{R}=2,4,6 \ldots & \longrightarrow & \left(\Gamma_{R \rightarrow[V V]_{2}}-\Gamma_{R \rightarrow[V V]_{0}}\right) \gtreqless 0, \\
\Gamma_{R \rightarrow V V}=\Gamma_{R \rightarrow[V V]_{2}} \geq 0, \quad \Gamma_{R \rightarrow[V V]_{0}}=0 & \text { for } J_{R}=3,5,7 \ldots & \longrightarrow & \left(\Gamma_{R \rightarrow[V V]_{2}}-\Gamma_{R \rightarrow[V V]_{0}}\right) \geq 0,
\end{array}
$$

On-shell resonances with $J_{R}=1$ are forbidden by the Landau-Yang theorem [22] and those with $J_{R}=2,4,6 \ldots$ can in principle decay into $[V V]_{0}$ and $[V V]_{2}$ states [19]. In QCD the $\gamma \gamma$ decay of the lowest-lying spin-2 resonances $\left(\mathcal{T}=a_{2}, f_{2}, f_{2}^{\prime}\right)$ predominantly occurs with helicity $\Delta \lambda=2$ [23], i.e., $\Gamma_{\mathcal{T} \rightarrow \gamma \gamma} \approx \Gamma_{\mathcal{T} \rightarrow[\gamma \gamma]_{2}}$. The sum-rule (10) is mostly saturated by the lightest $J_{R}=0\left(\pi^{0}, \eta, \eta^{\prime}\right)$ and $J_{R}=2$ $\left(a_{2}, f_{2}, f_{2}^{\prime}\right)$ meson multiplets [20, 21]: the large spin-2 positive contribution cancels out to a large extent the large negative spin- 0 contribution. Similar thing happens in the case of (spin-2) massive gravitons $G$ [9-12], where the decay $G \rightarrow V(\lambda) V\left(\lambda^{\prime}\right)$ always occurs with $\Delta \lambda=2$ as the graviton couples to the stress-energy tensor of the gauge field $V=\gamma, g^{a}$.

\section{Sum-rule with a large-width scalar: you can't just put it alone}

In this and next Sections, we will explore the FSR constraints on any possible BSM physics that shows up in the $\gamma \gamma$ channel at LHC or future colliders. Let us assume the existence of a diphoton scalar (or pseudoscalar) resonance $\mathcal{S}$ similar to the now discarded $750 \mathrm{GeV}$ candidate $[1,2,13]$. We will take the latter as a case of study to exemplify the analysis of future experimental signals.

Conveniently reordering the sum-rule (12) one has

$$
16 \pi \frac{\Gamma_{\mathcal{S} \rightarrow V V}}{M_{\mathcal{S}}^{3}}=\sum_{R \neq \mathcal{S}} 16 \pi\left(2 J_{R}+1\right) \frac{\left(\Gamma_{R \rightarrow[V V]_{2}}-\Gamma_{R \rightarrow[V V]_{0}}\right)}{M_{R}^{3}}+\text { non-R. }
$$

In the case of a large $\Gamma_{\mathcal{S} \rightarrow V V}$ partial width, resonances with $\Gamma_{R \rightarrow[V V]_{2}} \neq 0$ are needed on the r.h.s. to fulfill the identity (14), i.e., resonances with spin $J_{R} \geq 2$. This implies the existence of a infinite tower of higher spin resonances à la Regge [8] to cure the divergent high-energy behaviour of the cross channel resonance exchanges in the partial wave amplitudes [15]. These BSM resonance theory is non-renormalizable and dual to an underlying strongly coupled theory where the resonances are composite states.

This argumentation relies to a large extent on the fact that the $\Gamma_{\mathcal{S} \rightarrow V V}$ partial decay width is large, with

$$
16 \pi \frac{\Gamma_{\mathcal{S} \rightarrow V V}}{M_{\mathcal{S}}^{3}} \sim 1 \mathrm{TeV}^{-2},
$$


as it occurred with the former scalar candidate with mass $M_{\mathcal{S}} \sim 750 \mathrm{GeV}$ and a partial width $\Gamma_{\mathcal{S} \rightarrow V V} \sim$ $10 \mathrm{GeV}$ (for $V=\gamma, g^{a}$ ). In this situation size matters, as we find very difficult that the FSR (14) can be compensated by the non-resonant loop contributions to $V(k) V\left(k^{\prime}\right) \rightarrow V(k) V\left(k^{\prime}\right)$. Based on naive dimensional analysis, these are found to be small, of the order of

$$
\left.\frac{1}{\pi} \int_{v_{\text {th }}}^{\infty} \frac{\mathrm{d} v^{\prime}}{\left(v^{\prime}\right)^{2}} \operatorname{Im} T_{\Delta \lambda}\left(v^{\prime}+i \epsilon\right)\right|_{\text {non }-\mathrm{R}} \sim \frac{\alpha^{2}}{v_{\text {th }}} \sim 10^{-4} \mathrm{TeV}^{-2},
$$

where, for possible new physics states in the non-R loop in an underlying weakly interacting theory (if any), we expect the thresholds to be $v_{\text {th }}>(750 \mathrm{GeV})^{2}$. More precisely, in Quantum Electrodynamics (QED) with either a scalar or a spin- $\frac{1}{2}$ particle with charge $Q=1$, the $\gamma \gamma$ cross-section difference reaches a sharp global minimum with $\sigma_{2}(v)-\sigma_{0}(v) \gtrsim-8 \alpha^{2} / v_{\text {th }}$ right after the production threshold due to the negative $\Delta \lambda=0$ contribution, then a wider global maximum with $\sigma_{\Delta \lambda}(v) \lesssim 2 \alpha^{2} / v_{\text {th }}$ due to positive $\Delta \lambda=2$ production, and finally a converging $1 / v$ tail [21]. Thus, one finds that the pure QED one-loop amplitude for $\gamma \gamma \rightarrow \gamma \gamma$ fulfills the sum-rule (10) on its own and yields no correction to our FSR [21]. Therefore, in order to get a contribution from these background loops to cancel the scalar resonance one in Eq. (14), one should incorporate effects beyond QED, which first enter at two loops. We find very unlikely that these corrections are large enough to achieve this goal without entering a non-perturbative regime.

In the case of a small partial width $\Gamma_{\mathcal{S} \rightarrow V V}$, one does not need to include resonances with $J_{R} \geq 2$ and the sum-rule can be fulfilled through the non-resonant loop terms in (14). In that situation, the underlying theory is perturbative. For instance, in the SM, the Higgs exchange yields a much smaller contribution $16 \pi \Gamma_{h \rightarrow \gamma \gamma} / m_{h}^{3} \simeq 2.4 \times 10^{-4} \mathrm{TeV}^{-2}$ [24], which is cancelled out by the $\gamma \gamma \rightarrow \gamma \gamma$ loop amplitude without any need of BSM physics. For radiatively generated $\mathcal{S} \rightarrow V V$ decays $(V V=$ $\gamma \gamma, g g$ ) in weakly interacting theories in the $\mathrm{TeV}$, small partial widths are expected: one would have $\Gamma_{\mathcal{S} \rightarrow g g} \lesssim \alpha_{S}^{2} M_{\mathcal{S}}^{3} /\left(72 \pi^{3} v^{2}\right) \sim 10^{-5} \mathrm{TeV}$ for a Higgs-like decay $\mathcal{S} \rightarrow g g$ [25, 26], yielding a contribution to (12) of the order of $16 \pi \Gamma_{\mathcal{S} \rightarrow g g} / M_{\mathcal{S}}^{3} \leq 2 \alpha_{S}^{2} /\left(9 \pi^{2} v^{2}\right) \sim 10^{-3} \mathrm{TeV}^{-2}$. These numbers get roughly two orders of magnitude smaller for $\mathcal{S} \rightarrow \gamma \gamma$, for a similar Higgs-like radiatively generated decay [26]. A perturbative BSM theory including a scalar resonance with a loop-induced decay similar to the SM one needs either a large number of particles running in the intermediate loop or huge hypercharges [27] to give a contribution to (14) of the order of $1 \mathrm{TeV}^{-2}$. Achieving the latter and the required large background non-resonant contribution to the sum-rule implies a departure from perturbativity in the $\mathrm{TeV}$ range, where the BSM theory would enter a strongly coupled regime (see e.g. Ref. [7]). Thus, one would expect to have composite states of any total angular momenta $J_{R} \geq 2$ lying in the nonperturbative energy range, as nothing forbids excitations with an arbitrary orbital momentum, similar to what one observes in QCD.

\section{$4 J_{R}=0$ and $J_{R}=2$ resonances and $\gamma \gamma$ production at LHC}

Let us assume that the lightest and lowest-spin resonances dominate the FSR sum-rule. This means that the lightest scalar $\mathcal{S}$ and tensor $\mathcal{T}$ resonance partial widths are related in the approximate form

$$
\frac{16 \pi \Gamma_{\mathcal{S} \rightarrow V V}}{M_{\mathcal{S}}^{3}} \approx \frac{80 \pi\left(\Gamma_{\mathcal{T} \rightarrow[V V]_{2}}-\Gamma_{\mathcal{T} \rightarrow[V V]_{0}}\right)}{M_{\mathcal{T}}^{3}} \leq \frac{80 \pi \Gamma_{\mathcal{T} \rightarrow V V}}{M_{\mathcal{T}}^{3}} .
$$

If $\mathcal{T}$ only decays into helicity $\Delta \lambda=2$ states - as it happens in the case of massive gravitons [9-12]Eq. (17) turns into an identity and one obtains the lowest possible bound for $\Gamma_{\mathcal{T} \rightarrow V V}$. We will assume this lowest signal scenario $\Gamma_{\mathcal{T} \rightarrow V V}=\Gamma_{\mathcal{T} \rightarrow[V V]_{2}}$ from here on and assume the relation

$$
\Gamma_{\mathcal{T} \rightarrow V V} \approx \frac{\Gamma_{\mathcal{S} \rightarrow V V}}{5}\left(\frac{M_{\mathcal{T}}}{M_{\mathcal{S}}}\right)^{3}
$$


To illustrate how this relation may provide useful information about the diphoton data we will exemplify the analysis with the former $750 \mathrm{GeV}$ candidate being our scalar $\mathcal{S}$ and the lightest tensor $\mathcal{T}$ being provided by the $2.8 \sigma(2.4 \sigma)$ excess at $M_{\mathcal{T}} \approx 1.6 \mathrm{TeV}$ from ATLAS data presented in Moriond 2016 [2] (in August 2016 in ICHEP [13]). Using these numbers and a large partial width $\Gamma_{\mathcal{S} \rightarrow V V} \approx 10 \mathrm{GeV}$ as reference values one obtains

$$
\Gamma_{\mathcal{T} \rightarrow V V} \approx 20 \mathrm{GeV} \quad\left(\frac{\Gamma_{\mathcal{S} \rightarrow V V}}{10 \mathrm{GeV}}\right) \quad\left(\frac{0.75 \mathrm{TeV}}{M_{\mathcal{S}}}\right)^{3} \quad\left(\frac{M_{\mathcal{T}}}{1.6 \mathrm{TeV}}\right)^{3} .
$$

Assuming the inverted case $M_{\mathcal{T}} \approx 750 \mathrm{GeV}$ and $M_{\mathcal{S}} \approx 1.6 \mathrm{TeV}$, with $\Gamma_{\mathcal{T}(750) \rightarrow V V} \sim 10 \mathrm{GeV}$ would have led to a huge partial width $\Gamma_{\mathcal{S}(1600) \rightarrow V V} \sim 0.5 \mathrm{TeV}$ and a far too large and broad signal.

One can make a final exercise with our illustrative study with $M_{\mathcal{S}}=750 \mathrm{GeV}$ and $M_{\mathcal{T}}=1.6 \mathrm{TeV}$. Early 2016 combined analyses of the LHC13 diphoton data yielded a cross section $\sigma(p p \rightarrow \mathcal{S}(750) \rightarrow$ $\gamma \gamma)=(4.2 \pm 2) \mathrm{fb}$ [3], with ATLAS13 data pushing for higher values and CMS13 for a lower cross section. The best fit to the $3.2 \mathrm{fb}^{-1} 2015$ ATLAS13 (the $2.7 \mathrm{fb}^{-1}$ CMS13 data) pointed out an expected excess of 6.6 signal events ( 8 signal events) [3]. If $g g$ was the main was the main production channels one had

$$
\sigma(p p \rightarrow R)=\frac{\left(2 J_{R}+1\right) C_{g g}\left(M_{R}\right) \Gamma_{R \rightarrow g g}}{s M_{R}}, \quad \sigma(p p \rightarrow R \rightarrow \gamma \gamma)=\sigma(p p \rightarrow R) \mathcal{B}_{R \rightarrow \gamma \gamma},
$$

with $\sqrt{s}=8(13) \mathrm{TeV}[3,27]$. Adding the $\gamma \gamma$ fusion $[5,6]$ contribution does not change this picture. In the case of lepton colliders the latter channel might be the dominant production mode and one should make then the replacement $C_{g g}\left(M_{R}\right)$ and $C_{g g}\left(M_{R}\right) \Gamma_{R \rightarrow g g}$ by $C_{\gamma \gamma}\left(M_{R}\right)$ and $C_{\gamma \gamma}\left(M_{R}\right) \Gamma_{R \rightarrow \gamma \gamma}$, respectively, in the expressions for the cross sections and the tensor-to-scalar ratios in this Section. The different partonic contributions $\left(C_{g g}, C_{u u}, \ldots\right)$ can be found in Ref. [27], also including their (partondependent) scaling between 8 and $13 \mathrm{TeV}$ collision energies corresponding to LHC run-I and run-II data, respectively. Larger energies and higher-order corrections increase the relative importance of the $g g$ contribution. The ratio of tensor-to-scalar production cross section is given by

$$
\frac{\sigma(p p \rightarrow \mathcal{T})}{\sigma(p p \rightarrow \mathcal{S})}=\frac{5 C_{g g}\left(M_{\mathcal{T}}\right)}{C_{g g}\left(M_{\mathcal{S}}\right)} \frac{\Gamma_{\mathcal{T} \rightarrow g g} / M_{\mathcal{T}}}{\Gamma_{\mathcal{S} \rightarrow g g} / M_{\mathcal{S}}} \approx \frac{C_{g g}\left(M_{\mathcal{T}}\right)}{C_{g g}\left(M_{\mathcal{S}}\right)}\left(\frac{M_{\mathcal{T}}}{M_{\mathcal{S}}}\right)^{2} .
$$

For the values $M_{\mathcal{S}}=750 \mathrm{GeV}$ and $M_{\mathcal{T}}=1.6 \mathrm{TeV}$, an estimate through MG5_aMC [28] with the parton distribution function set NN23LO1 [29] yields

$$
\frac{C_{g g}\left(M_{\mathcal{T}}\right)}{C_{g g}\left(M_{\mathcal{S}}\right)}\left(\frac{M_{\mathcal{T}}}{M_{\mathcal{S}}}\right)^{2}=7.0 \%
$$

for $\sqrt{s}=13 \mathrm{TeV}$ and roughly a factor of two smaller for $\sqrt{s}=8 \mathrm{TeV}$. Assuming that $\mathcal{T} \rightarrow \gamma \gamma$ and $\mathcal{S} \rightarrow \gamma \gamma$ decays have a similar branching ratio -which is implied by (18) when $\gamma \gamma$ and $g g$ are the main decay channels- one expects the tensor-to-scalar ratio

$$
\frac{\sigma(p p \rightarrow \mathcal{T})}{\sigma(p p \rightarrow \mathcal{S})} \approx \frac{0.29 \mathrm{fb}}{4.2 \mathrm{fb}}
$$

Considering a detection efficiency for $\mathcal{T}(1600)$ similar to that obtained for the $\mathcal{S}(750)$ one it is possible to perform a rough estimate of the number of resonant diphoton events $N_{p p \rightarrow \mathcal{T} \rightarrow \gamma \gamma}$ that should have been observed for an integrated luminosity of $15 \mathrm{fb}^{-1}$ at each detector:

$$
N_{p p \rightarrow \mathcal{T} \rightarrow \gamma \gamma} \approx 2.2 \text { signal events (ATLAS 13), } \quad N_{p p \rightarrow \mathcal{T} \rightarrow \gamma \gamma} \approx 3.1 \text { signal events (CMS 13), }
$$


with the SM background being much smaller than these numbers at $1.6 \mathrm{TeV}$. Thus, joining both experiments, we should have observed around 5 events by August 2016. Unfortunately, at the same time that the significance of the $750 \mathrm{GeV}$ resonance faded away no clear signal could be observed for 1.6 TeV diphoton excess.

\section{Conclusions}

In this talk we have presented the work [14], where we showed that a colourless neutral scalar with a large $g g$ or $\gamma \gamma$ partial width cannot show up alone at LHC or future colliders. Our FSR analysis implies that either an infinite tower of resonance with spin $J_{R} \geq 2$ à la Regge must be present in the underlying theory, or this enters a non-perturbative regime at the $\mathrm{TeV}$-triggering the appearance of composite states and resulting in similar conclusions-.

Even though the $750 \mathrm{GeV}$ diphoton resonance has essentially disappeared back into the background after August 2016 LHC13 data, we have exemplified in these proceedings the kind of constraints that it is possible to extract with our FSR. Spin-2 (or higher) resonances are required to cancel out the scalar contribution to the sum-rule in the case of large partial width $\Gamma_{\mathcal{S} \rightarrow V V} \sim 10 \mathrm{GeV}$. Taking, the other higher significant excess in the data at $1.6 \mathrm{TeV}$ as our tensor candidate we were able to perform an estimate of how the $\mathcal{T}(1600)$ excess should also appear in following runs.

This powerful model-independent approach is based on basic principles such as analyticity, unitarity and crossing symmetry and can be easily applied in future diphoton analyses in case a new resonant signal appears. It may serve to pin down the necessary accompanying BSM states in the case of large partial widths $\Gamma_{R \rightarrow V V}$ which cannot be compensated in the FSR through perturbative loop contributions of a weakly interacting BSM theory.

Even in the case of small partial-width resonance, the FSR may prove to be useful, as the possible resonance excess generates an unbalance in the sum-rule that must be compensated. However, in that case, in addition to further resonances one should look for an excess in the background as the sumrule may now be compensated through the non-resonant loop diagrams of a weakly-interacting BSM theory. This task may be more cumbersome but still worthy to be analyzed in order to confirm or discard any possible new diphoton resonance.

\section{Acknowledgements}

PR acknowledges funding from Conacyt, México, through projects 296 ('Fronteras de la Ciencia'), 236394, 250628 ('Ciencia Básica') and SNI. The work of JJSC was supported by the Spanish Ministry MINECO under grant FPA2013-44773-P and MINECO:FPA2014-53375-C2-1-P.

\section{References}

[1] Tech. Rep. ATLAS-CONF-2015-081, CERN, Geneva, Dec, 2015. CMS Collaboration, collisions at $13 \mathrm{TeV}, \mathrm{CMS}-\mathrm{PAS}-\mathrm{EXO}-15-004 ; 16-018$.

[2] Talks given by M. Delmastro and P. Musella on behalf of the ATLAS and CMS Collaborations, respectively. To be published in the proceedings of the 51st Rencontres de Moriond EW, La Thuile, 12-19 March, 2016.

[3] J. S. Kim, K. Rolbiecki and R. Ruiz de Austri, Eur. Phys. J. C 76 (2016) no.5, 251. doi:10.1140/epjc/s10052-016-4102-0 [arXiv:1512.06797 [hep-ph]].

[4] F. Staub et al., Eur. Phys. J. C 76 (2016) no.9, 516 [arXiv:1602.05581 [hep-ph]].

[5] C. Csaki, J. Hubisz and J. Terning, Phys. Rev. D 93 (2016) no.3, 035002. 
[6] C. Csaki, J. Hubisz, S. Lombardo and J. Terning, Phys. Rev. D 93 (2016) no.9, 095020.

[7] E. Bertuzzo, P. A. N. Machado and M. Taoso, Phys. Rev. D 94 (2016) no.11, 115006 [arXiv:1601.07508 [hep-ph]].

[8] G. Veneziano, Nuovo Cim. A 57 (1968) 190.

[9] L. Randall and R. Sundrum, Phys. Rev. Lett. 83 (1999) 3370 ; 83 (1999) 4690.

[10] N. Arkani-Hamed, S. Dimopoulos and G. R. Dvali, Phys. Lett. B 429 (1998) 263. H. Davoudiasl, J. L. Hewett and T. G. Rizzo, Phys. Rev. Lett. 84 (2000) 2080.

[11] M. Gouzevitch, A. Oliveira, J. Rojo, R. Rosenfeld, G. P. Salam and V. Sanz, JHEP 1307 (2013) 148.

[12] V. Sanz, arXiv:1603.05574 [hep-ph].

[13] Talks given by D. Charlton and T. Camporesi on behalf of the ATLAS and CMS Collaborations, respectively. To be published in the proceedings of the 38th International Conference on High Energy Physics, August 3-10, 2016, Chicago.

[14] P. Roig and J. J. Sanz-Cillero, Phys. Rev. D 94 (2016) no.9, 095021 doi:10.1103/PhysRevD.94.095021 [arXiv:1605.03831 [hep-ph]].

[15] B. R. Martin, D. Morgan and G. Shaw, "Pion Pion Interactions in Particle Physics", London 1976, 460p.

[16] M. Froissart, Phys. Rev. 123 (1961) 1053.

[17] P. Roy, Phys.Rev. D 9 (1974) 2631. S. Gerasimov and J. Moulin, Nucl.Phys. B 98 (1975) 349.

S.J. Brodsky and I. Schmidt, Phys.Lett. B 351 (1995) 344.

[18] E. Euler, Ann. Phys., Lpz. 26 (1936) 398; E. Euler and W. Heisenberg, Z. Phys. 98 (1936) 714.

[19] G. Panico, L. Vecchi and A. Wulzer, JHEP 1606 (2016) 184.

[20] V. Pascalutsa and M. Vanderhaeghen, Phys.Rev.Lett. 105 (2010) 201603.

[21] V. Pascalutsa, V. Pauk and M. Vanderhaeghen, Phys.Rev. D 85 (2012) 116001.

[22] L. D. Landau, Dokl. Akad. Nauk Ser. Fiz. 60 (1948) 207 (1948) [Phys. Abstracts A 52 (1949) 125]; C.N. Yang, Phys. Rev. 77 (1950) 242.

[23] M. R. Pennington, T. Mori, S. Uehara and Y. Watanabe, Eur. Phys. J. C 56 (2008) 1.

[24] K.A. Olive et al.(Particle Data Group), Chin. Phys. C, 38, 090001 (2014) and 2015 update.

[25] F. Wilczek, Phys. Rev. Lett. 39 (1977) 1304.

[26] A. Djouadi, Phys. Rept. 457 (2008) 1.

[27] R. Franceschini et al., JHEP 1603 (2016) 144.

[28] J. Alwall et al., JHEP 1407 (2014) 079 [arXiv:1405.0301 [hep-ph]].

[29] R.D. Ball et al. (NNPDF Collaboration), Nucl.Phys. B 877 (2013) 290 [arXiv:1308.0598 [hep$\mathrm{ph}]$. 